\title{
Cisplatin-based chemotherapy with or without bevacizumab for Chinese postmenopausal women with advanced cervical cancer: a retrospective observational study
}

Xiaoli He ${ }^{1+}$, Jun Liü ${ }^{2+}$, Li Xiao ${ }^{3}$, Mingdong Zhao ${ }^{4}$, Tingting Su${ }^{5}$, Tiejian Liu', Guowei Han ${ }^{7}$ and Yue Wang ${ }^{1 *}$

\begin{abstract}
Background: The purpose of this study was to assess the efficacy and safety of cisplatin-based chemotherapy with or without bevacizumab (BEV) in Chinese women with advanced cervical cancer (ACC).

Methods: For this observational study, we analysed the data of 316 Chinese women with ACC who were treated at the Henan provincial people's hospital between Jan 1, 2014, and Dec 31, 2018, with cisplatin-based chemotherapy plus BEV (CB) or cisplatin-based chemotherapy alone (CA) until disease progression, unacceptable toxicity, or death. The co-primary endpoints were overall survival (OS) and progression-free survival (PFS); the secondary endpoint was the occurrence of adverse events (AEs).

Results: A total of 264 patients with ACC were included in the assessment (CB, $n=130$ and $C A, n=134)$. At a median follow-up of 38 months (IQR 36-40), the median OS in the CB cohort was significantly longer than that in the CA cohort (hazard ratio [HR] 1.21,95\% confidence interval[CI] 1.14-1.73; $p=0.002$ ); additionally, the median PFS was 345 days (95\% Cl, 318-372) for CB and 261 days (95\% Cl, 165-357) for CA(HR 1.61, 95\% Cl 1.12-2.17; $p=0.000)$. Significant differences were noted between groups in terms of thrombosis/embolism, neutropenia, and febrile neutropenia.

Conclusions: In Chinese women with ACC, cisplatin-based chemotherapy plus BEV is associated with improved survival compared to cisplatin-based chemotherapy alone. This finding suggests a positive survival benefit of antiangiogenesis therapy in this population.
\end{abstract}

Keywords: Bevacizumab, Cervical cancer, Chemotherapy, Overall survival, Progression-free survival

\footnotetext{
* Correspondence: xjk0328@163.com

${ }^{\dagger}$ Xiaoli He and Jun Liu contributed equally to this work.

'Department of Gynaecology, Henan Provincial People's Hospital, People's

Hospital of Zhengzhou University, People's Hospital of Henan University, No.

7. Weiwu Road, Jinshui District, Zhengzhou 450000, China

Full list of author information is available at the end of the article
}

\section{$\triangle B M C$}

(c) The Author(s). 2020 Open Access This article is licensed under a Creative Commons Attribution 4.0 International License, which permits use, sharing, adaptation, distribution and reproduction in any medium or format, as long as you give appropriate credit to the original author(s) and the source, provide a link to the Creative Commons licence, and indicate if changes were made. The images or other third party material in this article are included in the article's Creative Commons licence, unless indicated otherwise in a credit line to the material. If material is not included in the article's Creative Commons licence and your intended use is not permitted by statutory regulation or exceeds the permitted use, you will need to obtain permission directly from the copyright holder. To view a copy of this licence, visit http://creativecommons.org/licenses/by/4.0/ The Creative Commons Public Domain Dedication waiver (http://creativecommons.org/publicdomain/zero/1.0/) applies to the data made available in this article, unless otherwise stated in a credit line to the data. 


\section{Background}

Advanced cervical cancer (ACC) is usually regarded as a devastating disease affecting women worldwide because of its association with increased morbidity and mortality [1-6]. The incidence of ACC and associated mortality in China have been increasing since 2005 [7]. Treatment of ACC continues to be a challenge, although early-stage cancers may potentially be cured with radical surgery [7-10]. Five-year survival rates for patients with ACC varied from 4 to $15 \%$ within different study cohorts [3, 11]. Although the clinical outcomes for such cases have improved, the optimal strategy for ACC is still debatable $[2,8,10,12]$.

For patients with ACC, cisplatin-based chemotherapy has been regarded as the standard processing scheme, as in previous reports [13-17]. Nevertheless, findings from the Gynecologic Oncology Group (GOG)-240 randomized phase III trial [18] indicated that the incorporation of bevacizumab (BEV) with chemotherapy for recurrent, persistent or metastatic cervical cancer markedly increased the survival benefit. A recent phase III trial [19] using a $2 \times 2$ factorial design that was conducted to verify whether chemotherapy with or without BEV improves overall survival (OS) in women with ACC showed proof-of-concept of the efficacy and tolerability of anti-angiogenesis therapy in ACC because the sustained benefit conferred by chemotherapy plus BEV was evidenced by separated survival curves. Furthermore, the prospective validation of pooled prognostic factors in patients with ACC treated with chemotherapy with or without $\mathrm{BEV}$ demonstrated that the benefit to undergoing BEV tended to be remarkable in the moderate- and high-risk subgroups $[19,20]$. Several studies $[11,18,21]$ have tried to assess whether BEV is independently associated with survival. Nevertheless, such studies have had small sample sizes, have included patients undergoing drug treatment without the stratification of outcomes, or failed to adjust for some approved confounders. Furthermore, data regarding Chinese women with ACC who were treated with BEV-containing chemotherapy are extremely limited.

With these limitations in mind, we aimed to confirm whether Chinese women with ACC who are undergoing cisplatin-based chemotherapy plus BEV had greater survival benefits than those receiving cisplatin-based chemotherapy alone from Henan provincial people's hospital from 2014 to 2018.

\section{Methods}

\section{Study design and patient eligibility}

Data for 350 postmenopausal women with ACC were identified and retrieved from the Henan Provincial People's Hospital from Jan 1, 2014, to Dec 31, 2018. All demographic, clinicopathological, treatment, and survival data were obtained by trained clinical reviewers from the medical charts and telephone interviews with patients. The main inclusion criteria were as follows: postmenopausal women without menstruation for 12 consecutive months [13]; aged 55-75 years; metastatic, persistent, and recurrent cervical carcinoma; a history of papillomavirus (HPV) infections; at least one measurable lesion assessed according to the Response Evaluation Criteria in Solid Tumours (RECIST) version 1.1; adequate haematological levels, hepatic function, bone marrow function and renal function, as reported [19, 20, 22]; a GOG performance status score of 0 (fully active) or 1 [18]. The main exclusion criteria were as follows: patients with severe organ failure; rectofistula and/or vesical fistula; uncontrolled metabolic dysfunction; deaths from treatment-independent hypertension, cardiovascular events, and pneumonia; non-healing wounds; tumours invading major blood vessels; a high risk of bleeding; a thromboembolism event; cerebrovascular accident or coma lasting more than $24 \mathrm{~h}$; delirious or otherwise cognitively impaired [23]; no or poor pretreatment image data; or inadequate medical records. The co-primary endpoints were OS and PFS; the secondary endpoint was the incidence of AEs.

\section{Study design and treatment}

A retrospective, single-centre study was conducted in which eligible patients received chemotherapy with or without BEV [18]. The intravenous chemotherapy regimen consisted of cisplatin (at a dose of $50 \mathrm{mg}$ per square metre of body surface area) plus paclitaxel (at a dose of $175 \mathrm{mg} / \mathrm{m} 2$ on day 1 ); the intravenous BEV regimen was a dose of $15 \mathrm{mg} / \mathrm{kg}$ on day 1 ; the regimens for chemotherapy plus BEV (CB) and chemotherapy alone (CA) were repeated at 21-day intervals, as described [18]. Treatment was performed until disease progression, withdrawal, unacceptable AEs, death, or if the patient had a complete response.

\section{Definitions of the descriptive variables}

ACC was defined as metastatic, persistent, or recurrent cervical carcinoma, which was confirmed by a central pathology laboratory and through clinical imaging evidence. The co-primary endpoint measures were survival curves for OS and PFS. OS was computed from the date of drug treatment to the date of either death from any cause or final follow-up; PFS was calculated from the date of drug treatment to the date of either progression or death from any cause. Progression was defined according to the modified RECIST criteria. Disease assessment by contrast-enhanced computed tomography (CT) or magnetic resonance imaging (MRI) was performed at least every other month, regardless of patients exhibiting disease progression or not. Details related to disease 
assessment and tumour measurements were assessed according to the RECIST guidelines version 1.1 [24]. Safety assessment, which was performed according to the US national cancer institute's patient-reported outcomes version of the common terminology criteria for adverse events (PRO-CTCAE) [25] during each cycle, was performed at least every 2 weeks for the initial 12 weeks and repeated at least every 4 weeks until disease progression, withdrawal, unacceptable AEs, death, or if the patient had a complete response. BEV could be delayed or discontinued based on the occurrence, duration, and severity of AEs.

\section{Statistical analysis}

Baseline variables, treatment histories, and dates of first administration of drugs were obtained, along with dates of progression, final follow-up, and survival. We used Fisher's exact or $\chi^{2}$ tests for categorical variables and the Mann-Whitney U test for continuous variables. Survival analysis was estimated with the Kaplan-Meier approach, regardless of the duration or type of therapy received. The secondary endpoint measure was occurrence of AEs. Patients with missing data were excluded from the final analysis. The median follow-up was calculated by the reverse Kaplan-Meier approach. Hazard ratios (HRs) were estimated using a Cox proportional hazard model with a 95\% Wald confidence interval (95\% CI) [18]. All $p$ values were two-sided with the level of significance set at $<0.05$. We performed data management and analyses with SPSS version 24.0 (IBM, Inc., NY, USA).

\section{Results}

\section{Baseline characteristics}

We analysed retrospective data from 350 patients with ACC, of whom 264 patients $(\mathrm{CB}, n=130$, mean age 67.22 years [SD 5.33] and CA, $n=134,67.43$ years [SD 7.53]) were identified for study eligibility (Fig. 1). Baseline characteristics, which were well balanced between both cohorts, are summarized in Table 1 . The median follow-up for both cohorts was 38 months (IQR 36-40).

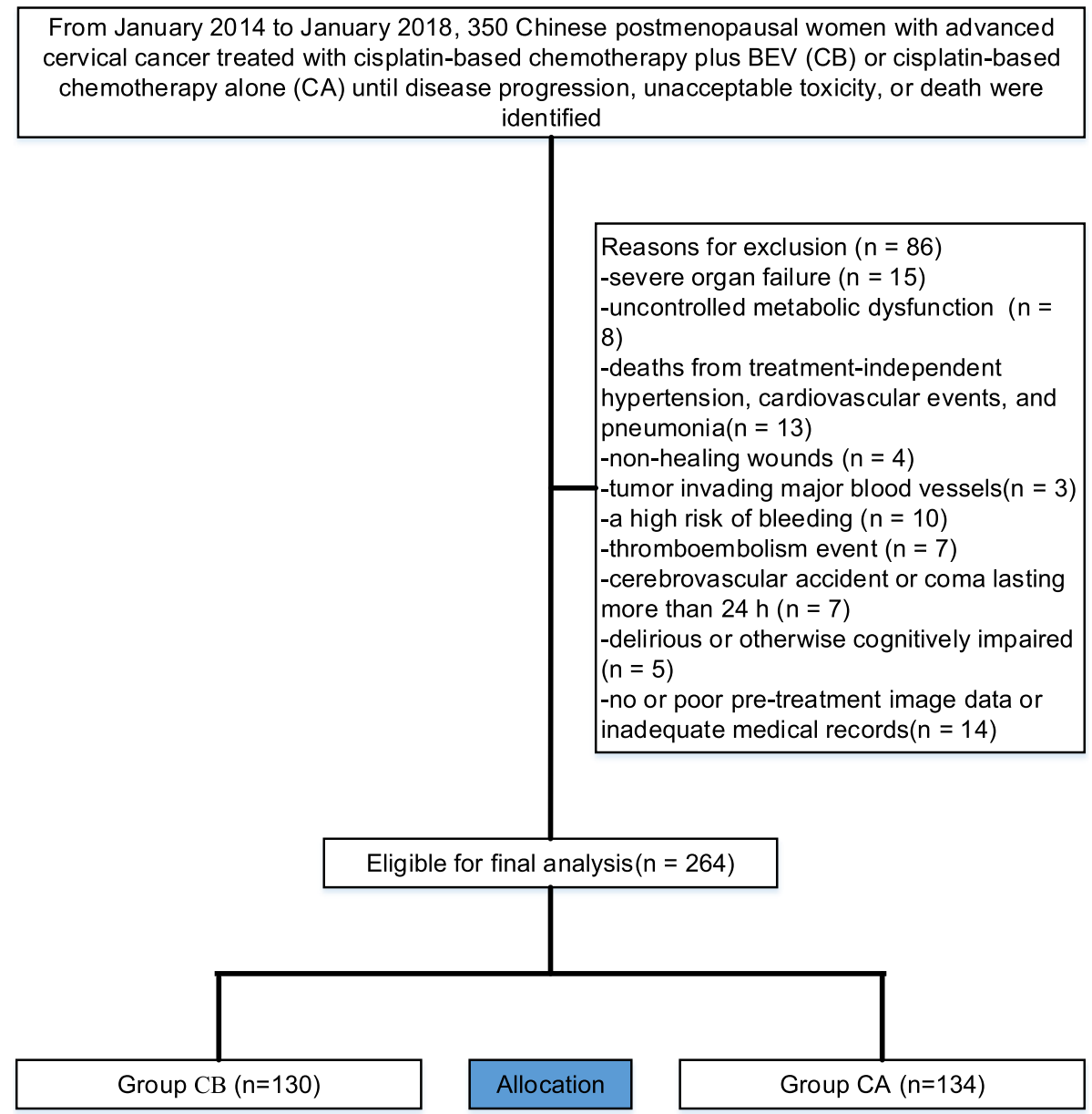

Fig. 1 Flow diagram demonstrating the methods used to identify studies to retrospectively evaluate the efficacy and safety of cisplatin-based chemotherapy with or without bevacizumab (BEV) in Chinese women with advanced cervical cancer (ACC) 
Table 1 Patient demographics between groups

\begin{tabular}{|c|c|c|c|}
\hline Variable & CB $(n=130)$ & CA $(n=134)$ & $p$-value \\
\hline Age at onset (years) & $67.22 \pm 5.33$ & $67.43 \pm 7.53$ & $0.346^{a}$ \\
\hline Histology, n (\%) & & & $0.885^{b}$ \\
\hline Squamous & $74(57)$ & $76(57)$ & \\
\hline Adenocarcinoma & $43(33)$ & $48(36)$ & \\
\hline Other & $13(10)$ & $10(7)$ & \\
\hline Disease presentation, n (\%) & & & $0.501^{b}$ \\
\hline Recurrent & $35(27)$ & $33(25)$ & \\
\hline Persistent & $27(21)$ & 25(19) & \\
\hline Stage IVB & $68(52)$ & $76(56)$ & \\
\hline Prior treatment, n (\%) & & & $0.489^{b}$ \\
\hline Radical surgery & $14(11)$ & $22(16)$ & \\
\hline Radical radiotherapy & 25(19) & $27(20)$ & \\
\hline Radiotherapy adjuvant & $34(26)$ & $30(22)$ & \\
\hline Radical chemoradiotherapy & 19(15) & $16(12)$ & \\
\hline Palliative radiotherapy & $27(21)$ & $23(17)$ & \\
\hline No prior treatment & $11(8)$ & $16(13)$ & \\
\hline Duration of treatment (mo) & $28.63 \pm 7.15$ & $28.35 \pm 7.42$ & $0.225^{a}$ \\
\hline Performance status (ECOG), n (\%) & & & $0.955^{b}$ \\
\hline 0 & $47(36)$ & $48(36)$ & \\
\hline 1 & $83(64)$ & $86(64)$ & \\
\hline GOG performance status, n (\%) & & & $0.970^{b}$ \\
\hline 0 & $42(32)$ & $43(32)$ & \\
\hline 1 & $88(68)$ & $91(68)$ & \\
\hline Number of metastatic sites, n (\%) & & & $0.234^{b}$ \\
\hline 3 & $33(25)$ & $27(20)$ & \\
\hline$>3$ & $77(59)$ & $81(60)$ & \\
\hline unknown & 20(16) & $26(20)$ & \\
\hline Prior pelvic radiotherapy, n (\%) & & & $0.889^{b}$ \\
\hline Yes & $60(46)$ & $63(47)$ & \\
\hline No & $70(54)$ & $71(53)$ & \\
\hline
\end{tabular}

${ }^{a}$ Analysed using an Independent-Samples t-test; ${ }^{b}$ Analysed using the MannWhitney $U$ test. $C B$ cisplatin-based chemotherapy plus bevacizumab, $C A$ cisplatin-based chemotherapy alone, GOG Gynecologic Oncology Group, ECOG Eastern Collaborative Oncology Group

The median number of cycles was 9 (range, 1-34) for $\mathrm{CB}$ and 10 (range, 1-39) for CA. Two hundred and twenty-eight $(86 \%)$ patients discontinued the intervention, mainly attributed to disease progression (45\% for $C B$ vs. $33 \%$ for $C A)$ and adverse events $(21 \%$ for $C B$ vs. $13 \%$ for CA). At final follow-up, sixteen patients that initially had tumour infiltration developed rectofistula and/ or vesical fistula(5[3.8\%] for CB vs. $11[8.2 \%]$ for $\mathrm{CA}, p=$ 0.137).

\section{Comparison of efficacy}

Final analysis of patient response showed that approximately $56 \%$ of patients responded on cisplatin-based chemotherapy with and without BEV. For the CBtreated cohort, the median OS was reached (95\% CI 18.0 months to not reached); the 1-year OS has not been reached; the 2-year OS was $45 \%(41-52)$. For the CAtreated cohort, the median OS was also reached (95\% CI 11.9 months to not reached); the 1-year OS has not been reached; the 2-year OS was 38\% (34-42). At final followup, the median OS was 540 days (95\% CI, 483-597) in the CB group and 357 days (95\% CI, 264-450) in the CA group; the median PFS was 345 days (95\% CI, 318-372) in the CB group and 261 days (95\% CI, 165-357) in the CA group. Significant differences were observed between groups in both the median OS (HR 1.21, 95\% CI 1.141.73; $p=0.002$ ) (Fig. 2) and median PFS (HR 1.61, 95\% CI $1.12-2.17 ; p=0.000$ ) (Fig. 3).

\section{Comparison of safety}

The main drug-related AEs, regardless of the study drug relationship, are summarized in Table 2. CA tends to be safer when patients have neutropenia based on the toxicity profile compared to that of $\mathrm{CB}$. The frequency and severity of drug-related AEs were in line with the acknowledged safety profile. Grade 3 or higher thromboembolism was more frequent in the $\mathrm{CB}$ group than in the CA group (13.1\% vs $5.2 \%$; $p=0.026)$. Neutropenia or febrile neutropenia occurred in 35 cases (13.3\%) among both groups, of which 9 occurred during the first 4 months in the CA group and 26 occurred during the first 7 months in the CB group. Additionally, AEs leading to dose interruptions or permanent discontinuation occurred in 37 cases (24 [18.5\%] for CB-treated cohort and 13 [9.7\%] for CA-treated cohort; $p=0.040$ ), primarily owing to grade $\geq 3$ neutropenia or thrombosis/embolism. Other grade 2 or higher AEs were fistula (occurring in 30 cases [11.4\%]), hypertension (in 32 cases [12.1\%]), bleeding (in 17 cases [6.4\%]), proteinuria (in 26 cases [9.8\%]), and pain (in 53 cases [20.1\%]), which were not significantly different between groups. The incidence of other AEs of special interest (i.e., headache, emesis) was generally similar between the groups.

\section{Discussion}

To the best of our knowledge, this study is the largest so far on postmenopausal Chinese women with ACC who were treated with cisplatin-based chemotherapy with or without BEV. Our study met its co-primary endpoints; the BEV-containing regimen was associated with an increased survival benefit. The superiority of $\mathrm{CB}$ over $\mathrm{CA}$ in this setting tended to be positive. BEV-related AEs were similar to those observed in previous reports.

Several limitations should be considered. First, the retrospective nature of our analysis with this methodology decreased the power to draw reliable conclusions, and some potential variables (such as some medical 


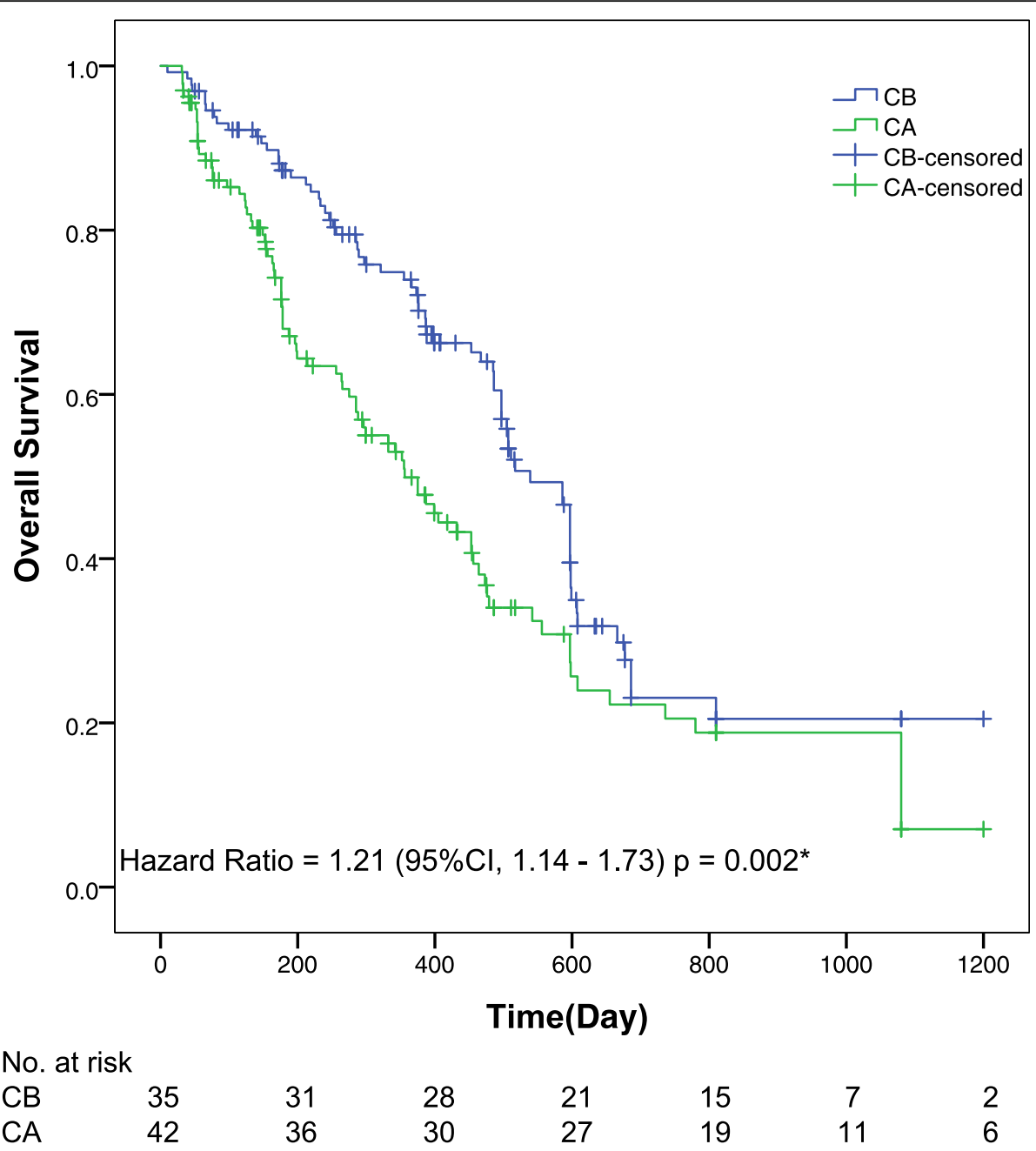

Fig. 2 Kaplan-Meier curves for overall survival. The median overall survival was 18.0 months (95\% confidence interval [CI], 16.1-19.9) and 11.9 months (95\% Cl, 8.8-15.0) for the CB and CA groups, respectively. Significant differences were detected in overall survival between groups. ${ }^{*}$ The hazard ratio was calculated using a Cox proportional hazards model, with the age, the site of primary tumour, the number of metastatic sites, and the performance status used as covariates and CB/CA therapy as the time-dependent factor. With respect to the overall survival, the results of a log-rank test reported $p=0.002$

diseases) could not be addressed in our analysis. Second, the relatively small sample size in the present study may have introduced bias. Third, generalizability was lacking owing to the study population involving only Chinese postmenopausal patients with ACC. Fourth, power might be underestimated, primarily due to our analysis involving repeated observations of each subject.

Our analysis determined survival with a longer followup and was consistent with previous findings $[11,13,18]$ that $\mathrm{CB}$ improves survival benefit in patients with ACC, since the 3-year OS reported here $(41 \%)$ is similar to that reported in a randomised, controlled, open-label, phase 3 trial (39\%) [19]. In view of multiple regimens with noteworthy activity in ACC treatment, clinical OS results might be confounded by the availability of these regimens [18]. BEV, a humanized anti-VEGF monoclonal antibody, has already demonstrated remarkable activity in ACC, as assessed by response rate [11, 19]; however, the effect of BEV on survival benefit needs to be determined as an indication of definitive survival benefit [13]. Survival benefit has conventionally been considered the most dependable endpoint in assessing cancer-related treatments [18, 20, 21].

In a phase III randomized trial [19] using a $2 \times 2$ factorial design, 452 ACC patients from 164 institutions in the United States and Spain were enrolled and randomized to receive $\mathrm{CB}$ or $\mathrm{CA}$ and showed significant improvement in OS: 16.8 vs 13.3 months for the $\mathrm{CB}$ and CA groups, respectively (HR, 0.77; 95\% CI, 0.62-0.95; $p=0.0068$ ), and PFS also favoured BEV (HR 0.68; 95\% CI $0.56-0.84 ; p=0.0002$ ). Additionally, a recent retrospective study [11] demonstrated a survival benefit of 


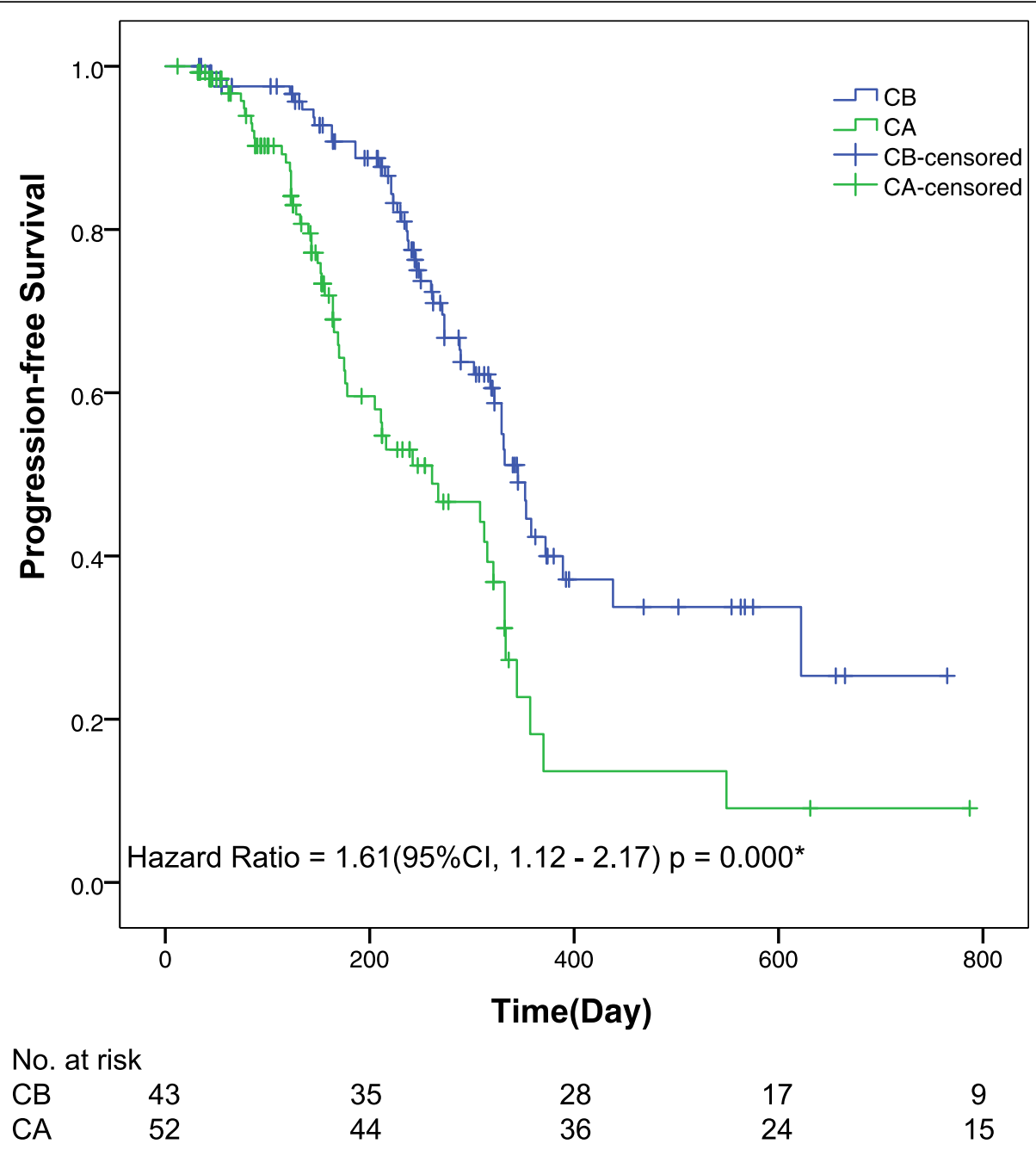

Fig. 3 Kaplan-Meier curves for progression-free survival. The median progression-free survival was 11.5 months ( $95 \%$ confidence interval [Cl], 10.6-12.4) and 8.7 months ( $95 \% \mathrm{Cl}, 5.5-11.9)$ for the CB and CA groups, respectively. Statistically significant differences were observed in progression-free survival between groups. *The hazard ratio was calculated using a Cox proportional hazards model, with age, the site of primary tumour, the number of metastatic sites, and the performance status used as covariates and CB/CA therapy as the time-dependent factor. With respect to progression-free survival, the results of a log-rank test reported $p=0.000$

Table 2 Comparison of the incidence of main drug-related AEs of grade $\geq 2$ between the groups at final follow-up

\begin{tabular}{llll}
\hline AEs & CB $(n=130)$ & CA $(n=134)$ & $p$-value \\
\hline Thrombosis/embolism, grade $\geq 3, \mathrm{n}(\%)$ & $17(13.1)$ & $7(5.2)$ & $3(2.2)$ \\
Neutropenia, grade $\geq 4, \mathrm{n}(\%)$ & $10(7.7)$ & $6(4.5)$ & $0.026^{* a}$ \\
Febrile neutropenia, grade $\geq 3, \mathrm{n}(\%)$ & $16(12.3)$ & $9(6.7)$ & $0.021^{\text {*a }^{\mathrm{a}}}$ \\
Fistula, grade $\geq 2, \mathrm{n}(\%)$ & $8(6.2)$ & $6(4.5)$ & $0.852^{\mathrm{a}}$ \\
Fistula, grade $\geq 3, \mathrm{n}(\%)$ & $7(5.4)$ & $17(12.7)$ & $0.733^{\mathrm{a}}$ \\
Hypertension, grade $\geq 2, \mathrm{n}(\%)$ & $15(11.5)$ & $8(6.0)$ & $0.775^{\mathrm{a}}$ \\
Bleeding, grade $\geq 3, \mathrm{n}(\%)$ & $9(6.9)$ & $15(11.2)$ & $0.752^{\mathrm{a}}$ \\
Proteinuria, grade $\geq 3, \mathrm{n}(\%)$ & $11(8.5)$ & $28(20.9)$ & $0.456^{\mathrm{a}}$ \\
Pain, grade $\geq 2, \mathrm{n}(\%)$ & $25(19.2)$ & $0.736^{\mathrm{a}}$ \\
\hline
\end{tabular}

${ }^{*}$ Statistically significant values. ${ }^{a}$ Analysed using the chi-square test. $A E s$ adverse events, $C B$ cisplatin-based chemotherapy plus bevacizumab, $C A$ cisplatin-based chemotherapy alone, AEs adverse events 
BEV when combined with chemotherapy in patients with recurrent, persistent or advanced cervical cancer. Why these analogous treatment regimens translated into corresponding gains in survival benefit is not confounding. In the current study, the large effect of $\mathrm{CB}$ on the treatment of ACC in the first 1 year with little effect thereafter was interesting.

Although chemotherapy plus BEV has been confirmed in patients with ACC in previous trials, data in the patient population remain limited $[18,20]$. Recently, a randomized trial by Penson [21] assigned 390 evaluable ACC patients to analyse patient reported outcomes in GOG 240 and showed that CB significantly improves OS, PFS, and response rates compared to CA. In the ACC setting, it is important to evaluate any lengthening in the duration of PFS and OS. Nevertheless, frequent debate often occurs regarding the influence of the oestrogen, predominantly in the postmenopausal cohort [26-28]. To reduce the impact of oestrogen on survival in the present study, the primary strategy was to only include a postmenopausal cohort. For these individuals who were ineligible for radical resection but still have their disease confined to the uterus, uterus-directed therapies may play a prominent role in reducing tumour burden and increasing survival [28]. However, for patients with ACC, chemotherapy plus BEV might be a good choice that has exhibited a positive impact on survival $[19,21]$.

There remains a paucity of survival data in the previous trials of postmenopausal patients with ACC [11, 13, 18]. Although it recognizes a distinct separation of PFS and OS curves, favouring the continuation of BEV, the continuation of $\mathrm{BEV}$ beyond progression failed to produce promising outcomes [18]. Moreover, the absolute improvements in the survival benefit appear small for this postmenopausal cohort with ACC and tended to be in association with the timing of tumour assessments $[29,30]$. In contrast to previous studies $[18,19,21]$, nevertheless, further analysis showed no considerable interaction between the continuation of $\mathrm{BEV}$ beyond progression and survival benefit.

\section{Conclusion}

The results reported here support the growing body of evidence that cisplatin-based chemotherapy plus BEV conferred a significant survival benefit versus cisplatinbased chemotherapy alone for Chinese women with ACC. In light of this finding, we are currently advocating for the incorporation of $\mathrm{BEV}$ with cisplatin-based chemotherapy as a clinical decision in this patient population. Future trials regarding the efficacy and safety of cisplatin-based chemotherapy plus BEV in a similar setting are needed.

\section{Abbreviations}

BEV: Bevacizumab; ACC: Advanced cervical cancer; CB: Cisplatin-based chemotherapy plus BEV; CA: Cisplatin-based chemotherapy alone; OS: Overall survival; PFS: Progression-free survival; AEs: Adverse events; HR: Hazard ratio; Cl: Confidence interval; GOG: Gynecologic Oncology Group; RECIST: Response Evaluation Criteria in Solid Tumours; CT: Computed tomography; MRI: Magnetic resonance imaging; PRO-CTCAE: Patient-reported outcomes version of the common terminology criteria for adverse events; IQR: Interquartile range; SD: Standard deviation

\section{Acknowledgments}

Not applicable.

\section{Authors' contributions}

$\mathrm{XH}$ : Planning and study design, study execution, and writing-initial draft. JL: Statistical analysis/interpretation, and writing-final revision. LX and MZ: Planning and study design. TS, TL and GH: Data collection and study execution. YW: Writing-initial draft, and writing-final revision. All authors have read and approve the final version.

\section{Funding}

This work was supported by grants from the Shanghai Municipal Health and Family Planning.

Commission (2014-403 and 201640057). All the grand supporters have no role in the design of the study and collection, analysis, and interpretation of data and in writing the manuscript.

\section{Availability of data and materials}

The datasets used and/or analysed during the current study are available from the corresponding author on reasonable request.

\section{Ethics approval and consent to participate}

The retrospective study was approved by the institutional review board of Henan Provincial People's Hospital and written informed consent was obtained for each patient prior to sample collection. Patients were informed that the resected specimens were stored by the hospital and potentially used for scientific research, and that their privacy would be maintained.

\section{Consent for publication}

Not applicable.

\section{Competing interests}

The authors declare that they have no competing interests.

\section{Author details}

'Department of Gynaecology, Henan Provincial People's Hospital, People's Hospital of Zhengzhou University, People's Hospital of Henan University, No. 7, Weiwu Road, Jinshui District, Zhengzhou 450000, China. ${ }^{2}$ Department of Gynaecology, The First Affiliated Hospital, Sun Yat-sen University, No. 58, Zhongshan 2nd Road, Yuexiu District, Guangzhou 510080, China.

${ }^{3}$ Department of Gynaecology and Obstetrics, The Central Hospital of Wuhan, Tongji Medical College, Huazhong University of Science and Technology, No. 26, Shengli Street, Jiang'an District, Wuhan 430014, Hubei, China.

${ }^{4}$ Department of Orthopaedics, Jinshan Hospital, Fudan University, Longhang Road No. 1508, Jinshan District, Shanghai 201508, China. ${ }^{5}$ Department of Gynaecology, The Obstetrics and Gynecology Hospital of Fudan University, No. 419, Fangxie Road, Huangpu District, Shanghai 200011, China.

${ }^{6}$ Department of Neurosurgery, The Third Affiliated Hospital of Southern Medical University, No.183, Zhongshan avenue west, Tianhe District, Guangzhou 510630, China. ${ }^{7}$ Department of Orthopaedics, The First Affiliated Hospital, Sun Yat-sen University, No. 58, Zhongshan 2nd Road, Yuexiu District, Guangzhou 510080, China.

Received: 31 May 2019 Accepted: 12 April 2020

Published online: 05 May 2020

\section{References}

1. Minion LE, Bai JR, Monk BJ, Keller LR, Ramez EN, Forde GK, et al. A Markov model to evaluate cost-effectiveness of antiangiogenesis therapy using bevacizumab in advanced cervical cancer. Gynecol Oncol. 2015;137(3):490-6. 
2. Fagotti A, Conte C, Stollagli F, Gallotta V, Salutari V, Bottoni C, et al. Radical surgery in advanced cervical Cancer patients receiving Bevacizumabcontaining chemotherapy a "real life experience". Int J Gynecol Cancer. 2018:28(8):1569-75.

3. Arbyn M, Castellsague X, de Sanjose S, Bruni L, Saraiya M, Bray F, et al. Worldwide burden of cervical cancer in 2008. Ann Oncol. 2011;22(12):2675-86.

4. Gupta S, Maheshwari A, Parab P, Mahantshetty U, Hawaldar R, Sastri S, et al. Neoadjuvant Chemotherapy Followed by Radical Surgery Versus Concomitant Chemotherapy and Radiotherapy in Patients With Stage IB2, IIA, or IIB Squamous Cervical Cancer: A Randomized Controlled Trial. J Clin Oncol. 2018;36(16):1548.

5. Stevanovic S, Draper LM, Langhan MM, Campbell TE, Kwong ML, Wunderlich JR, et al. Complete Regression of Metastatic Cervical Cancer After Treatment With Human Papillomavirus-Targeted Tumor-Infiltrating T Cells. J Clin Oncol. 2015;33(14):1543.

6. Vale C, Tierney JF, Stewart LA, Brady M, Dinshaw K, Jakobsen A, et al. Reducing uncertainties about the effects of Chemoradiotherapy for cervical Cancer: a systematic review and meta-analysis of individual patient data from 18 randomized trials. J Clin Oncol. 2008;26(35):5802-12.

7. Zhang J, Yao TT, Wang YX, Yu J, Liu YY, Lin ZQ. Long noncoding RNA MEG3 is downregulated in cervical cancer and affects cell proliferation and apoptosis by regulating miR-21. Cancer Biol Ther. 2016;17(1):104-13.

8. Koh WJ, Greer BE, Abu-Rustum NR, Apte SM, Campos SM, Cho KR, et al. Cervical Cancer, version 2.2015 featured updates to the NCCN guidelines. J Natl Compr Cancer Netw. 2015;13(4):395-404.

9. Marth C, Landoni F, Mahner S, McCormack M, Gonzalez-Martin A, Colombo $\mathrm{N}$, et al. Cervical cancer: ESMO clinical practice guidelines for diagnosis, treatment and follow-up. Ann Oncol. 2017;28:72-83.

10. Katki HA, Kinney WK, Fetterman B, Lorey T, Poitras NE, Cheung $L$, et al. Cervical cancer risk for women undergoing concurrent testing for human papillomavirus and cervical cytology: a population-based study in routine clinical practice. Lancet Oncol. 2011;12(7):663-72.

11. Godoy-Ortiz A, Plata Y, Alcaide J, Galeote A, Pajares B, Saez E, et al. Bevacizumab for recurrent, persistent or advanced cervical cancer: reproducibility of GOG 240 study results in "real world" patients. Clin Trans Oncol. 2018:20(7):922-7.

12. Small W, Bacon MA, Bajaj A, Chuang LT, Fisher BJ, Harkenrider MM, et al. Cervical Cancer: a Global Health crisis. Cancer. 2017:123(13):2404-12.

13. Tamimi RM, Hankinson SE, Chen WY, Rosner B, Colditz GA. Combined estrogen and testosterone use and risk of breast cancer in postmenopausal women. Arch Intern Med. 2006;166:1483-9.

14. Mitsuhashi A, Uno T, Usui H, Tate S, Hirashiki K, Kato K, et al. Postoperative concurrent daily low-dose Cisplatin-based Chemoradiation improves the prognosis of patients with pathologic T2b or N1 cervical Cancer. Anticancer Res. 2010;30(6):2341-6.

15. Rose PG, Lappas PT. Analysis of the cost effectiveness of concurrent cisplatin-based chemoradiation in cervical cancer: implications from five randomized trials. Gynecol Oncol. 2000;78(1):3-6.

16. Martinez-Fernandez MI, Folgueira JL, Peydro GV, Cambeiro M, Espinos J, Aramendia JM, et al. Long-term results of a phase II trial of concomitant Cisplatin-paclitaxel Chemoradiation in locally advanced cervical Cancer. Int J Gynecol Cancer. 2016;26(6):1162-8.

17. Yao TT, Lu RB, Zhang J, Fang XY, Fan L. Huang CX, et al. Growth arrestspecific 5 attenuates cisplatin-induced apoptosis in cervical cancer by regulating STAT3 signaling via miR-21. J Cell Physiol. 2019;234(6):9605-15.

18. Tewari KS, Sill MW, Long HJ, Penson RT, Huang H, Ramondetta LM, et al. Improved survival with Bevacizumab in advanced cervical Cancer. N Engl J Med. 2014;370(8):734-43.

19. Tewari KS, Sill MW, Penson RT, Huang $H$, Ramondetta LM, Landrum LM, et al. Bevacizumab for advanced cervical cancer: final overall survival and adverse event analysis of a randomised, controlled, open-label, phase 3 trial (gynecologic oncology group 240). Lancet. 2017;390(10103):1654-63.

20. Tewari KS, Sill MW, Monk BJ, Penson RT, Long HJ, Poveda A, et al. Prospective validation of pooled prognostic factors in women with advanced cervical Cancer treated with chemotherapy with/without Bevacizumab: NRG oncology/ GOG study. Clin Cancer Res. 2015;21 (24):5480-7.

21. Penson RT, Huang HQ, Wenzel LB, Monk BJ, Stockman S, Long HJ, et al. Bevacizumab for advanced cervical cancer: patient-reported outcomes of a randomised, phase 3 trial (NRG oncology-gynecologic oncology group protocol 240). Lancet Oncol. 2015;16(3):301-11.
22. Qiao YL, Sellors JW, Eder PS, Bao YP, Lim JM, Zhao FH, et al. A new HPVDNA test for cervical-cancer screening in developing regions: a crosssectional study of clinical accuracy in rural China. Lancet Oncol. 2008;9(10): 929-36.

23. Alvarez-Sabin J, Ortega G, Jacas C, Santamarina E, Maisterra O, Ribo M, et al. Long-term treatment with Citicoline may improve Poststroke vascular cognitive impairment. Cerebrovasc Dis. 2013;35(2):146-54.

24. Edeline J, Boucher E, Rolland $Y$, Vauleon E, Pracht M, Perrin C, et al. Comparison of tumor response by response evaluation criteria in solid tumors (RECIST) and modified RECIST in patients treated with sorafenib for hepatocellular carcinoma. Cancer. 2012;118(1):147-56.

25. Dueck AC, Mendoza TR, Mitchell SA, Reeve BB, Castro KM, Rogak LJ, et al. Validity and reliability of the US National Cancer Institute's patient-reported outcomes version of the common terminology criteria for adverse events (PRO-CTCAE). JAMA Oncol. 2015;1 (8):1051-9.

26. Frantsiyants E, Komarova E, Nikitina V, Spiridonova D, Cheryarina N. Estrogen metabolites, level of $E 7$, and squamous cell carcinoma autigenin the tissue of cervical cancer. J Clin Oncol. 2014;32(15).

27. Mori T, Sawada M, Kuroboshi H, Tatsumi H, Katsuyama M, Iwasaku K, et al. Estrogen-related receptor alpha expression and function are associated with vascular endothelial growth factor in human cervical Cancer. Int J Gynecol Cancer. 2011;21(4):609-15.

28. Ourmantcheeva A, Berstein L, Burnina M, Zeldovich D. Estrogen replacement therapy (ERT) in patients with cervical cancer. Ann Oncol. 2000; 11:88.

29. Kang S, Nam BH, Park JY, Seo SS, Ryu SY, Kim JW, et al. Risk assessment tool for distant recurrence after platinum-based concurrent Chemoradiation in patients with locally advanced cervical Cancer: a Korean gynecologic oncology group study. J Clin Oncol. 2012;30(19):2369-74.

30. Beavis AL, Gravitt PE, Rositch AF. Hysterectomy-corrected cervical Cancer mortality rates reveal a larger racial disparity in the United States. Cancer. 2017;123(6):1044-50

\section{Publisher's Note}

Springer Nature remains neutral with regard to jurisdictional claims in published maps and institutional affiliations.

Ready to submit your research? Choose BMC and benefit from

- fast, convenient online submission

- thorough peer review by experienced researchers in your field

- rapid publication on acceptance

- support for research data, including large and complex data types

- gold Open Access which fosters wider collaboration and increased citations

- maximum visibility for your research: over $100 \mathrm{M}$ website views per year

At BMC, research is always in progress.

Learn more biomedcentral.com/submissions 